DEM ONSTRATION NOUVELLE

DU THÉORĖME DE LAURENT

PAR

\title{
G. MITTAG-LEFFLER
}

a S TOCKHOLM.

Dans son célèbre mémoire: Zur Theorie der eindeutigen analytischen Functionen, Wererstrass a démontré le théorème suivant:

$\boldsymbol{A}$. DSoit $f(x)$ une fonction monogène uniforme de la variable $x$, possédant les $n$ points singuliers essentiels $c_{1}, \ldots, c_{n}$.

Il est toujours possible de représenter cette fonction sous la forme suivante:

On construit une fonction rationnelle du degré $n$ de la variable $x, y=\varphi(x)$, possédant les póles $c_{1}, \ldots, c_{n}$. On forme ensuite, ce qui est toujours possible, $n$ fonctions monogènes et uniformes de la variable $y, F_{0}(y), F_{1}(y), \ldots, F_{n-1}(y)$, n'ayant pas d'autre point singulier essentiel que $y=\infty$, constituant en outre des fonctions entières rationnelles ou transcendantes quand $f(x)$ ne possède pas de pôles, et étant enfin telles, que l'on a partont l'égalité

$$
f(x)=\sum_{y=0}^{n-1} F_{\nu}(y)\left(\frac{1}{x-c}\right)^{\nu},
$$

où la constante $c$ désigne l'une des $n$ valeurs $c_{1}, \ldots, c_{n}$.)

Si $c=\infty$, on entend, comme toujours chez Weifrstrass, par $x-\infty$ l'expression $\frac{1}{x}$. 
Ce théorème ne parait pas avoir été jusqu'ici l'objet de l'attention qu'il mérite à tous égards. La raison en est peut-être qu'il figure chez Weicistrass principalement comme théorème auxiliaire, servant à démontrer le théorème suivant:

$\boldsymbol{B}$. )Soit $f(x)$ une fonction monogène uniforme de la variable $x$, ayant pour points singuliers $c_{1}, \ldots, c_{n}$. Cette fonction peut toujours être représentée sous la forme

$$
f(x)=C+\sum_{\nu=1}^{n} G_{\nu}\left(\frac{1}{x-c_{\nu}}\right)
$$

où $C$ désigne une constante indépendante de $x$, et où $G_{\nu}\left(\frac{\mathrm{I}}{x-c_{\nu}}\right)$ est une fonction entière rationnelle ou transcendante de $\frac{\mathrm{I}}{x-a_{\nu}}$, s'évanouissant, lorsque $\frac{\mathrm{I}}{x-c_{\nu}}=\mathrm{O.H}$

On voit sans peine que le théorème $\boldsymbol{B}$ découle immédiatement du théorème qui suit, connu sous le nom du théorème de Laurent.

"Soit, pour $R^{\prime}<|x|<R^{\prime \prime}$, où l'on entend par $R^{\prime}$ et $R^{\prime \prime}$ des quantités positives données, $f(x)$ une fonction monogène, uniforme et régulière de la variable $x$. Il est toujours possible de constituer une série $\sum_{n=-\infty}^{n=+\infty} A_{n} x^{n}$, dont les coefficients sont indépendants de $x$, et qui est telle, en outre, que l'égalité

$$
f(x)=\sum_{\mu=-\infty}^{\mu=+\infty} A_{\mu} x^{\mu}
$$

a lieu partout pour $R^{\prime}<|x|<R^{\prime \prime}$.»

Le théorème de LaUnent se laisse facilement dériver de la théorie des intégrales définies, et il n'est alors qu'un simple corollaire d'un théorème de Cauchy.

Démontré de cette façon, le théorème de Launest ne reçoit cependant pas, dans la théorie des fonctions, la place élémentaire qui semble devoir lui appartenir.

Il paraît que par cette raison et afin de ne pas abandonner l'ordre d'idées auxquelles appartiennent les recherches consignées dans le mémoire: 
Zur Theorie der eindentigen analytischen Functionen, Weierstrass a déduit le théorème $\boldsymbol{B}$ du théorème $\boldsymbol{A}$, au lieu de passer par le théorème de Laurentr. $\left(^{(}\right) \mathrm{Si}$, comme je l'ai fait dans divers mémoires, on poursuit la voie ouverte par Weicrstrass, on constate cependant bientòt qu'il est impossible de se passer du théorème de Laurent. Il n'est toutefois pas nécessaire d'abandonner pour le reste les considérations élémentaires dont se sert Weierstrass.

Il y a donc une importance réelle à démontrer le théorème de LAURENT sans recourir au calcul intégral, et sans abandonner les éléments de la théorie des fonctions.

On peut obtenir cette démonstration en présentant le théorème $\boldsymbol{A}$ sous une autre forme que chez Weierstrass. Si l'on examine de plus près la méthode dont le grand géomètre se sert pour déduire le théorème $\boldsymbol{A}$, on voit sans peine qu'il a démontré en même temps le théorème suivant, qu'il n'énonce toutefois pas sous une forme explicite.

$C$. गSoit dans un continuum $\Re$, composé d'une seule pièce, $f(x)$ une fonction monogène uniforme, qui ne possède en $\mathfrak{x}$ aucun point singulier essentiel. Soit ensuite $y=\varphi(x)$ une fonction rationnclle du degré $n$ de la variable $x$, qui se comporte d'une façon régulière en dedans de $x$. Les valeurs de $y$ obtenues de l'égalité $y=\varphi(x)$, quand $x$ signifie successivement la totalité des points du continuum $\mathfrak{X}$, constituent, réunies, dans le domaine de la variable $y$, un continuum $\mathfrak{B}$ se composant d'une seule pièce. Supposons que $\mathfrak{X}$ et $\mathfrak{B}$ correspondent entre eux de façon que toutes les valeurs de $x$ satisfaisant à l'égalité $y=\varphi(x)$ pour une valeur donnée de $y$ située en dedans de $\mathfrak{B}$, soient elles-mèmes situées en dedans de $\boldsymbol{x}$.

Désignons par $c$ un point quelconque, situé sur la limite de $\mathfrak{x}$ ou en dehors de $\mathfrak{X}$. Il est toujours possible de construire $n$ fonctions $F_{\theta}(y), F_{1}(y), \ldots, F_{n-1}(y)$, qui constituent en $\not \mathcal{B}$ des fonctions monogènes uniformes de la variable $y$, ne possédant pas de point singulier essentiel, et pouvant être choisies de façon que l'égalité $f(x)=\sum_{\nu=0}^{n-1} F_{\nu}(y) \cdot \frac{1}{(x-c)^{\nu}}$ pour $y=\varphi(x)$, a lieu partout en dedans de $x$ et de 23 . Si la fonction 
Démonstration nouvelle du théorème de Laurent.

$f^{\prime}(x)$ n'a pas de póles dans le domaine $\mathfrak{x}$, il en est de mème, dans le domaine $\not 3$, de chacune des fonctions $\left.F_{0}(y), F_{1}(y), \ldots, F_{n-1}(y).\right)$

De ce théorème, celui de Laurent peut ètre déduit de la manière suivante.

Posons

$$
y=\frac{1}{2}\left[\left(\frac{x}{R}\right)^{n}+\left(\frac{k}{x}\right)^{n}\right]
$$

où $R$ signifiera une quantité positive donnée, et $n$ un nombre entier positif donné.

L'équation

$$
\frac{1}{2}\left[\left(\frac{x}{R}\right)^{n}+\left(\frac{R}{x}\right)^{n}\right]-y=\varphi(x)-y=0
$$

considérée par rapport à $x$, n'a de racines égales que dans le cas où $\varphi^{\prime}(x)=0$. Or, ce cas se présente toujours et exclusivement, lorsque $\left(\frac{x}{h}\right)^{n}= \pm \mathrm{I}$, et, par conséquent, lorsque $y= \pm \mathrm{I}$. Quand $y= \pm \mathrm{I}$ les différentes racines de l'équation $\varphi(x)-y=0$, se déduisent de l'expression $R . e^{\frac{2 k \pi i}{n}}$, en donnant $\dot{\mathrm{a}} k$ les valeurs successives $0, \mathrm{I}, 2, \ldots, n-1$. Chacune de ces racines est une racine double. Quand $y=-\mathrm{I}$, l'expression $R . e^{\frac{(2 k+1) \pi i}{n}}(k=0,1,2, \ldots, n-1)$ représente les racines différentes de l'équation $\varphi(x)-y=0$, et chacune de ces racines est aussi une racine double.

Donnons maintenant à $y$ une valeur finie $y^{\prime}$, qui n'est égale ni ̀̀ $+\mathrm{I}$, ni ̀̀ $-\mathrm{I}$. Si $x^{\prime}$ est une valeur correspondante de $x$, telle que $\varphi\left(x^{\prime}\right)-y^{\prime}=0$, l'égalité $\varphi(x)-y^{\prime}=0$ a lieu pour

$$
x=e^{\frac{2 k \pi i}{n}} \cdot x^{\prime}, \quad(i=0,1,2, \ldots, n, \cdots-1)
$$

et en outre pour

$$
x=e^{\frac{2 \xi \pi i}{n}} \cdot \frac{R^{2}}{x^{\prime}}
$$$$
\cdot(k=0,1,2, \ldots, n-1)
$$

Ces deux expressions donnent par conséquent les $2 n$ racines de l'égalité $\varphi(x)-y^{\prime}=0$. 
En faisant donc parcourir à $x$ toutes les valcurs remplissant la condition $\left|\frac{x}{R}\right|=\mathrm{I}$, ou étant situées sur la circonférence d'un cercle dans le plan de $x$ ayant lorigine pour centre et $R$ pour rayon, on verra $y$ parcourir simultanément toutes les valeurs réelles à partir de + I jusqu’i - I. Toutes les valeurs de $x$ qui répondent à une valeur réelle de $y$ telle que $-\mathrm{I} \leqq y \leqq+\mathrm{I}$ appartiennent aussi à la circonférence $\left|\frac{x}{R}\right|==\mathrm{I}$.

De même, si l'on fait parcourir à $x$ toutes les valeurs qui remplissent la condition $\left|\frac{x}{R}\right|=\mathbf{I}+\delta$, ò̀ $o$ est unc quantité positive donnée, et qui constituent par suite les points différents d'une circonférence daus le plan de $x$, dont le centre est à l'origine et dont le rayon est $\hat{R}(\mathrm{I}+\partial)$; $y$ parcourra simultanément dans le plan de y tous les points d'une courbe fermée, symétrique par rapport tant à l'axe des ordonnées qua l'axe des abscisses et limitant une surface simplement connexe, laquelle renferme $y=+\mathrm{I}$. Ia même courbe est aussi décrite par $y$ quand $x$ parcourt toutes les valeurs remplissant la condition $\left|\frac{R}{x}\right|=1+\delta$, et constituant par suite, dans le plan de $x$, les différents points d'une circonférence dont le centre est à l'origine et dont le rayon est $\frac{R}{i+\delta}$. D'un autre côté, à chaque point y appartenant à la courbe mentionnée du plan de $y$ correspondent $n$ points différents $x$, situés sur la circonférence ayant $R\left(\mathrm{I}+o^{\prime}\right)$ pour rayon, et $n$ autres points également différents les uns des autres, situés sur la circonférence ayant $\frac{R}{1+\hbar}$ pour rayon. On voit aussi que la plus grande distance de l'origine à un point de la courbe indiquée dans le plan de $y$ est

$$
\frac{1}{2}\left[(1+\delta)^{n}+\frac{1}{(1+\delta)^{n}}\right]
$$

et que la plus petite distance est $\frac{1}{02}\left[(1+\delta)^{n}-\frac{1}{(1+\delta)^{n}}\right]$.

Il suit de ce qui précède que, à l'anneau circulaire dans le plan de $x$ situé entre les deux circonférences $|x|=R(1+\rho)$ et $|x|=\frac{R}{1+\rho}$, si l'on entend par $\rho$ une quantité positive donnée, correspond, dans le plan de 
y, une surface simplement connexe, symétrique par rapport tant à l'axe des ordonnées quà l'axe des abscisses, surface enfermant le point $y=+\mathrm{I}$, et dont la ligne limite est telle, que la plus grande distance. entre un point de cette limite et l'origine est $\frac{1}{2}\left[(1+\rho)^{n}+\frac{1}{(1+\rho)^{n}}\right]$, et la plus petite distance est $\frac{\mathrm{I}}{2}\left[(\mathrm{I}+\rho)^{n}-\frac{\mathrm{I}}{(\mathrm{I}+\rho)^{n}}\right]$.

Désignons maintenant l'anneau circulaire par $\mathscr{X}$, et par $\mathfrak{3}$ la surface correspondante du plan de $y$. Tous les points du plan de $x$ correspondant à un point du plan de $y$, situé en dedaus de 3 , sont eux-mêmes toujours situés en dedans de $\mathfrak{X}$ ct tous les points correspondant à un point situé sur la limite de $: 3$ sont cux-ınémes situés sur la limite de 90 La fouction

$$
y=\frac{1}{2}\left[\left(\frac{x}{R}\right)^{n}+\left(\frac{R}{x}\right)^{n}\right]
$$

ne possède aussi que des points réguliers en dedans de $\mathfrak{H}$ et sur la limite

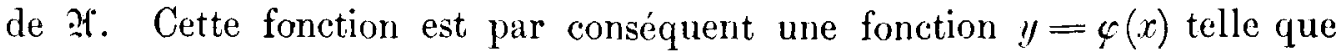
nous l'avons supposée dans le théorème $\boldsymbol{C}$.

La quantité $\dot{\rho}$ étant fixée, il est toujours possible de donner à $n$ une valeur assez grande pour que

$$
\frac{\mathrm{I}}{2}\left[(\mathrm{I}+\rho)^{n}-\frac{\mathrm{I}}{\left(\mathrm{I}+\boldsymbol{c}^{n}\right)^{n}} \mid>\mathrm{I}+h,\right.
$$

en désignant par $h$ une quantité positive quelconque. En admettant ensuite une quantité $\varepsilon$ assez petite pour que

$$
\frac{1}{2}\left[(1+\xi)^{n}+\frac{1}{(1+\xi)^{n}}\right]<1+h
$$

la surface qui dans le plan de $y$ répond à l'anncau circulaire

$$
\frac{R}{\mathrm{I}+\Sigma} \leqq|x| \leqq R(1+\leqq)
$$

est située en dedans d'un cercle qui a l'origine pour centre et i $+h$ pour rayon. 
Après ces remarques préliminaires, jobtiens facilement le théorème de Launent. Soit, pour toutes les valeurs de $x$ qui remplissent la condition $R^{\prime}<|x|<R^{\prime \prime}, f(x)$ une fonction monogène, uniforme et régulière de la variable $x$.

Fixons d'une manière arbitraire une quantité positive $R$ telle que $R^{\prime}<R<R^{\prime \prime}$. Posons ensuite une quantité positive $\rho$ telle que

$$
R(\mathrm{I}+\rho)<R^{\prime \prime} \text { et que } \frac{R}{\mathrm{I}+\rho}>R^{\prime}
$$

Désignons par $\mathfrak{X}$ l'anneau circulaire

$$
\frac{R}{\mathrm{1}+\rho}<|x|<R(\mathrm{1}+\rho)
$$

et par $h$ une quantité positive arbitraire. Choisissons le nömbre entier positif $n$ si grand que

$$
\frac{1}{2}\left[(\mathrm{I}+\rho)^{n}-\frac{\mathrm{I}}{(\mathrm{I}+\rho)^{n}}\right]>\mathrm{I}+h
$$

et indiquons par $\not 3$ le domaine de la variable $y$ qu'on déduit de l'égalité

$$
y=\frac{\mathrm{I}}{2}\left[\left(\frac{x}{k}\right)^{n}+\left(\frac{k}{x}\right)^{n}\right]
$$

en faisant parcourir $\dot{a} x$ le domaine $x$.

Conformément au théorème $\boldsymbol{C}$ on obtient alors l'égalité

$$
f(x)=\sum_{\nu=0}^{2 n-1} F_{y}(y)\left(\frac{1}{x-c}\right)^{\nu}
$$

où $c$ est un point en dedans ou sur la limite du domaine $\mathfrak{X}$ et oì les fonctions $F_{v}(x)$ en dedans du domaine $\boldsymbol{3}$ sont des fonctions monogénes, uniformes et régulières de la variable $y$. Le domaine 3 enferme totalement un cercle ayant l'origine pour centre et $\mathrm{I}+h$ pour rayon. On a donc pour ce cercle

$$
F_{\nu}(y)=A_{0}^{(\nu)}+A_{1}^{(\nu)} y+A_{2}^{(\nu)} y^{2}+\ldots,
$$


où $A_{0}^{(\nu)}, A_{1}^{(\nu)}, \ldots$ sont des constantes indépendantes de $y$ et $\sum_{\mu=0}^{\infty} A_{\mu}^{(\nu)}(\mathrm{I}+h)^{\mu}$ est une série à convergence absolue. Si $\varepsilon$ représente une quantité positive suffisamment petite pour que

$$
\frac{1}{2}\left[(I+\varepsilon)^{n}+\left(\frac{I}{I+\varepsilon}\right)^{n}\right]<I+h,
$$

le module de $y$ sera toujours inférieur à $\mathrm{I}+h$, dès que $x$ appartiendra à l'anneau circulaire $\frac{R}{\mathrm{I}+\varepsilon} \leqq|x| \leqq R(\mathrm{I}+\varepsilon)$. La série $\sum_{\mu=0}^{\infty} A_{\mu}^{(\nu)} \varphi(x)^{\mu}$, où

$$
\varphi(x)=\frac{\mathrm{I}}{2}\left[\left(\frac{x}{R}\right)^{n}+\left(\frac{R}{x}\right)^{n}\right]
$$

est par suite uniformément convergente pour toutes les valeurs de $x$ appartenant à l'anneau circulaire qui vient d'être mentionné. Ainsi par suite d'un théorème connu, (') on a dans cet anneau circulaire

$$
F_{\nu}(y)=\sum_{\mu=0}^{\infty} A_{\mu}^{(n)} \varphi(x)^{\mu}=G_{\nu}(x)+\bar{G}_{\nu}\left(\frac{1}{x}\right)
$$

oì $G_{\nu}^{\prime}(x)$ est une série procédant d'après les puissances positives de $x$, et convergente aussitót que $|x| \leqq R(1+\varepsilon)$, et $\bar{G}_{v}\left(\frac{1}{x}\right)$ est une série procédant d'après les puissances négatives de $x$, et convergente dès que $|x| \geqq \frac{R}{I+\varepsilon}$.

Vu l'égalité

$$
f(x)=\sum_{\nu=0}^{2 n-1} F_{\nu}(y) \cdot\left(\frac{1}{x-c}\right)^{\nu}
$$

on aura donc, pour l'anneau circulaire $\frac{R}{1+\varepsilon} \leqq|x| \leqq R(1+\varepsilon)$, l'égalité

$$
f(x)=\sum_{\mu=-\infty}^{\mu=+\infty} A_{\mu} x^{\mu}
$$

(') K. WeInestass: Zur Funktionenlehre. Monatsbericht der Königl. Akademie der Wissenschaften zu Berlin, August 1880 , p. 7 . 
Or il est évident, d'un côté, que l'égalité ci-dessus aura lieu partout à l'intérieur de l'anneau circulaire constituant le domaine de convergence commun des séries $\sum_{\mu=1}^{\infty} A_{-n} x^{-\gamma}$ et $\sum_{n=0}^{\infty} A_{n} x^{\mu}$, et, de l'autre, que ces deux séries doivent converger dans l'anneau, en dedans duquel $f(x)$ est une fonction monogène, uniforme et régulière de $x$. L'égalité

$$
f(x)=\sum_{\mu=-\infty}^{\mu=+\infty} A_{\mu} x^{\mu}
$$

a donc lieu non seulement pour le domaine $\frac{R}{\mathrm{I}+\varepsilon} \leqq|x| \leqq R(\mathrm{I}+\varepsilon)$, mais aussi pour le domaine $R^{\prime}<|x|<R^{\prime \prime}$. Le théorène de LAUrEv'T se trouve ainsi complètement démontré. 